\title{
Uma Abordagem Baseada em Perfil e Recomendação para Relatar Problemas de Infraestrutura no contexto de Smart Campus
}

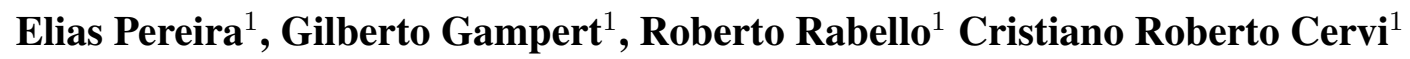 \\ ${ }^{1}$ Instituto de Ciências Exatas e Geociências (Iceg) \\ PPGCA - Programa de Pós-Graduação em Computação Aplicada \\ Universidade de Passo Fundo (UPF) - Passo Fundo - RS - Brazil \\ $\{177706$, gampert, rabello, cervi\}@upf.br
}

\begin{abstract}
A smart campus refers to a campus where modern information and communication technologies bring more benefits to life on campus, help users to improve and perform daily activities more efficiently, and improve social interactions. This article aims to present an approach based on personalization and recommendation, developed for mobile devices, which provides opportunities to users the pointing of infrastructure problems in the smart campus context, monitoring the development of problem solving, as well as interaction with users who reported the same problems.
\end{abstract}

Resumo. Um smart campus remete a um campus onde modernas tecnologias da informação e comunicação trazem mais vantagens à vida no campus, ajudam os usuários a melhorar e realizar suas atividades diárias de forma mais eficiente, além de melhorar as interações sociais. Esse artigo tem por objetivo apresentar uma abordagem baseada em personalização e recomendação, desenvolvida para dispositivos móveis, que oportuniza a usuários o apontamento de problemas de infraestrutura no contexto de smart campus, o acompanhamento da evolução da solução de problemas, bem como a interação com usuários que relataram os mesmos problemas.

\section{Introdução}

À medida que o mundo se prepara para a quarta revolução industrial que irá remodelar a maneira como vivemos, o conceito de cidades inteligentes surge como parte integrante dessa revolução. A partir deste conceito, foi desenvolvido um novo conceito de smart campus, que leva em conta indicadores de desempenho e características, desenvolvendo o campus através do uso eficiente de recursos, aplicando diversos tipos de inteligência, a fim de fornecer serviços inteligentes e de alta qualidade para as comunidades do campus, reduzindo custos operacionais e tornando a vida mais fácil e melhor. [Muhamad et al. 2017]. A característica relevante para esta pesquisa é o intelligent environment que destaca a maneira como as pessoas interagem com o ambiente e a maneira como o campus usa e gerencia seus recursos.

Para isso, propõe-se uma abordagem baseada no uso combinado de reputação e recomendação que oportuniza ao usuário relatar problemas de infraestrutura em seu campus, bem como a interação com uma Entidade Gestora que faz o monitoramento e o 
acompanhamento dos problemas relatados. Trata-se de um aplicativo para dispositivos móveis que possibilita ao cidadão enviar os relatos, e um Portal de Gestão que permite a Entidade Gestora monitorar e interagir com estes relatos.

O artigo está dividido como segue. A seção 2 apresenta os conteúdos estudados para a fundamentação teórica. Na seção 3 a abordagem proposta é especificada. Os experimentos e a análise dos resultados são apresentados e discutidos na seção 4. A seção 5 apresenta as considerações finais. Por fim, são apresentadas as referências bibliográficas utilizadas para o desenvolvimento do artigo.

\section{Referencial Teórico}

Esta seção apresenta uma visão geral sobre o estado da arte desta pesquisa especificando conceitos, domínios, tecnologias e ferramentas aplicadas ao tema Cidades Inteligentes com a abordagem em smart campus. Também são apresentados conceitos de perfil de usuários e sistemas de recomendação, pois são temas essenciais ao contexto deste artigo.

\subsection{Cidades Inteligentes}

Segundo [Flôr and Teixeira 2018], ao utilizar o termo de cidades inteligentes se faz referência a uma cidade funcional e em equilíbrio, em que a relação dos seus cidadãos, suas necessidades básicas e mais complexas são satisfeitas por meio da conexão de diversos mecanismos. Nesse sentido, [Eremia et al. 2017] afirmam que uma cidade inteligente é aquela que consegue fazer a união de infraestrutura física e legal, por meio das tecnologias de informação e comunicação (TICs), para que ocorra um equilíbrio entre a economia, o social e o meio ambiente.

Para [Capdevila and Zarlenga 2015], os principais desafios para um projeto de cidade inteligente são: (i) desenvolvimento econômico; (ii) inclusão social; (iii) segurança; (iv) sustentabilidade; (v) infraestrutura; (vi) transporte; (vii) moradia. As novas tecnologias de informação e comunicação permitiram a democratização da capacidade de inclusão dos cidadãos, que passaram a ter o poder de participar da dinâmica de inovação de suas cidades. Nessa leitura, a cidade inteligente deve ter uma forte abordagem orientada para a governança que enfatize o papel do capital social e das relações no desenvolvimento urbano Albino et al. 2015.

Apesar de alguns domínios serem distintos, existem aqueles que coincidem nas propostas estudadas. Isto se deve ao fato que cada cidade inteligente possui suas particularidades, e seus domínios variam de acordo com as suas necessidades e prioridades.

\subsection{Smart Campus e suas tecnologias}

Um smart campus é semelhante à cidades inteligentes e as mesmas soluções podem ser usadas em aplicativos de smart campus. O intuito dos Smart campuses é ser construído para o beneficio de professores, alunos e demais integrantes do campus, gerenciar os recursos disponíveis e melhorar a experiência do usuário com serviços proativos [Yu et al. 2011]. Esses exemplos incluem; renovação de infra-estruturas tecnológicas, enriquecimento de tecnologia e métodos de ensino, a tecnologia que permite a comunicação contínua entre estudantes e acadêmicos [Hirsch and Ng 2011], [Abuelyaman 2008].

Em outras palavras, o conceito de smart campus é um refinamento do termo inteligente intelligent environment, definido como um ambiente físico onde as TICs inovadoras 
e abrangentes permitem que as pessoas vivenciem e interajam com o espaço e os dados gerados [Prandi et al. 2019].

Nesses intelligent environments, o papel dos usuários está se tornando cada vez mais relevante [Mirri et al. 2018], passando de beneficiários passivos de serviços para participantes ativos [Prandi et al. 2018], exploradores de dados e colaboradores, também por meio de suas atividades em mídia social [Tse et al. 2016]. Este é o contexto em que o conceito de dados hiperlocais surgiu como crucial para capacitar uma comunidade.

Nesse contexto, o protótipo de realidade aumentada [Özcan et al. 2017] desenvolvido na Universidade Mugla Sitki Kogman (MSKU) é uma grande iniciativa de tecnologias para smart campus. Métodos de detecção de imagem são usados para detectar os locais do campus. Fontes de dados externas, incluindo dados do Twitter, são utilizados para obter informações sobre os locais detectados e apresentá-los na tela.

O artigo de [Loriot et al. 2017] apresenta uma a análise do uso da tecnologia LoRaWan em larga escala no contexto de smart campus. De acordo com os autores, os dados são coletados principalmente de sensores inteligentes para medir parâmetros físicos, assim como para armazenar, analisar e trocar dados com outros sistemas.

\subsection{Modelagem e Perfil de Usuários}

O perfil do usuário é considerado como um conjunto de dados estruturados descrevendo o ambiente de interação entre um usuário e um sistema. No domínio dos mecanismos de busca na Internet [Calegari and Pasi 2010], o perfil do usuário é uma ontologia usada para ter uma representação estruturada dos interesses do usuário. Permite representar o conhecimento (contexto e preferências do usuário) como um conjunto de conceitos.

A coleta de informação pode ser: (i) Explícita: O usuário é solicitado a inserir uma certa quantidade de informações; (ii) Implícita: Tem como objetivo a coleta de informações silenciosa através da observação da sensibilidade do usuário a determinados documentos ou da análise de documentos em seu diretório pessoal. Essa abordagem se torna mais difícil, mas no entanto podem ajudar a coletar uma grande quantidade de informações sem que o usuário seja solicitado; (iii) híbrida: Tem como propósito modelar o perfil de um usuário dependendo do domínio de aplicação e do próprio usuário, uma vez que tem como premissa que o sistema deve ter a possibilidade de se adaptar diante do cenário de utilização.

\subsection{Sistemas de Recomendação}

Para [Lopes 2012], os sistemas de recomendação surgiram para prover uma interface alternativa para tecnologias de filtragem e recuperação de dados, com o objetivo de suprir o usuário com informações que fossem de seu interesse. Para que a recomendação ocorra, geralmente são utilizados três tipos de informações: descrição do item a ser recomendado, informação do usuário que receberá a recomendação e avaliação do item recomendado ao usuário. 


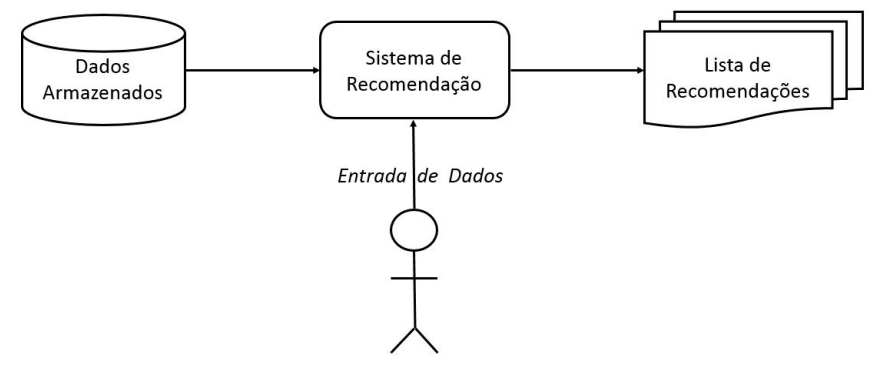

Figura 1. Visão geral de um sistema de recomendação.

Exemplos de aplicações do sistema de recomendação incluem sugestões para compras, serviços de saúde e atividades de entretenimento. Normalmente, um sistema de recomendação concentra-se em alguns itens específicos por exemplo, roupas, filmes ou livros para produzir sugestões úteis e eficazes para a seleção de itens [Abuein et al. 2017].

Em nossa abordagem, o conceito de recomendação se dá pelo encaminhamento do problema relatado pelos usuários ao setor mais competente para tratar de sua solução. Assim sendo, desenvolvemos um algoritmo que faz uso de uma base de problemas passados para recomendar a melhor solução de um novo problema que se apresenta.

\section{Materiais e Métodos}

Este seção apresenta um relato a respeito da arquitetura da abordagem proposta, bem como a descrição de tarefas principais do aplicativo e seus serviços. O projeto utilizou da abordagem de pesquisa híbrida, pois utiliza de dados matemáticos e de iteração com usuários para se chegar aos objetivos propostos.

\subsection{Arquitetura}

O app foi implementado utilizando a suite Android Studio e suas bibliotecas. No desenvolvimento do Painel de Gestão foram utilizadas as linguagens de programação $P H P$ e jQuery e os dados foram armazenados no banco de dados $M y S Q L$. Os dados e o portal que contém o Painel de Gestão utilizam o conceito de Computação em Nuvem. Na comunicação com o Portal de Gestão foram utilizados os conceitos de web services RESTful. Utilizando uma distribuição Linux Debian 8.4 como host para as aplicações e serviços.

A abordagem proposta permite que o usuário use um aplicativo móvel para realizar o relatório de problemas de infra-estrutura e interação com outros usuários que relatam o mesmo problema. Pode-se enriquecer as descrições com imagens, vídeo e posicionamento geográfico. Esses elementos contribuem para que o gerente possa acompanhar com mais qualidade os problemas e interação entre os usuários. 


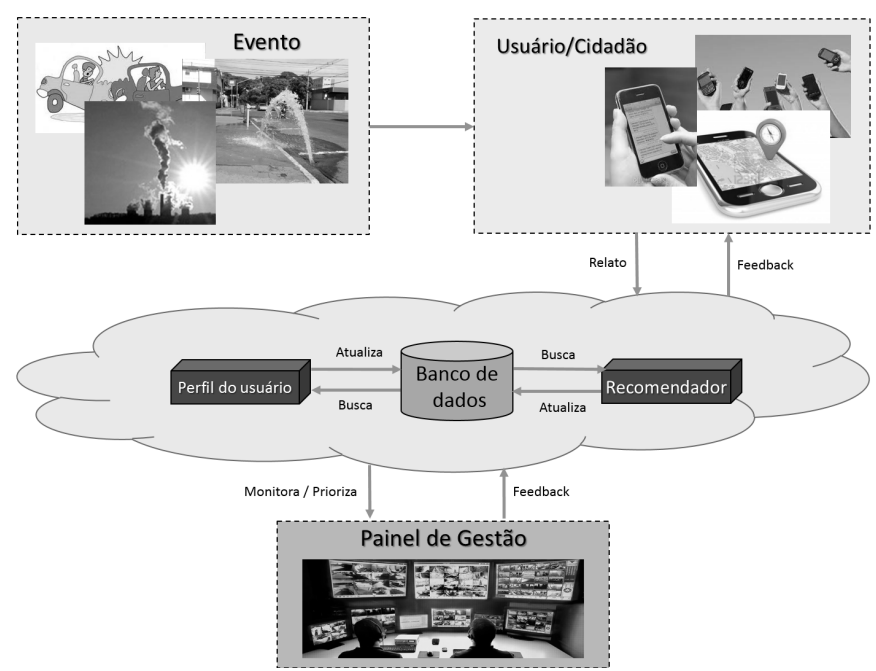

Figura 2. Arquitetura da abordagem proposta.

\subsection{Métrica da reputação e força dos relatos}

A fundamentação da reputação está ligada a um vetor de elementos, que leva em consideração a participação dos usuários e sua interação com o app. O calculo da reputação $R U V$ pode ser observado na equação (1).

$$
\mathrm{RUV}_{(\mathrm{u})}=\sum_{i=1}^{e_{i}} \frac{\left(v_{i} \cdot w_{i}\right)}{\max \left(v_{i}\right)}
$$

Onde $\left.R U V_{(} u\right)$ é a Reputação do app, $u$ identifica o usuário, $e_{i}$ é a quantidade de elementos que compõe a reputação, $i$ é a variação de 1 até $e_{i}, v_{i}$ é valor de cada elemento (interação, assertividade, resolução, colaboração e adesão), $w_{i}$ é o peso de cada elemento e $\max \left(v_{i}\right)$ é maior valor de cada elemento.

A Tabela 1 apresenta os elementos que definem o modelo de reputação e seus respectivos pesos. Foram atribuídos pesos maiores para os elementos Interação, Colaboração e Adesão tendo em vista obter uma maior participação dos usuários, e por consequência um maior empoderamento. 
Tabela 1. Elementos que compõe a reputação do usuário no app.

\begin{tabular}{l|l|c}
\hline Elemento & \multicolumn{1}{|c}{ Descrição } & Peso \\
\hline Interação & $\begin{array}{l}\text { A cada novo relato (problema) ou } \\
\text { interação do usuário representa um } \\
\text { aumento da reputação. }\end{array}$ & 30 \\
\hline Assertividade & $\begin{array}{l}\text { Se o relato (problema) do evento for } \\
\text { procedente (verdadeiro) a reputação } \\
\text { aumenta e, caso contrário a reputação } \\
\text { diminui. }\end{array}$ & 10 \\
\hline Resolução & $\begin{array}{l}\text { Se o Gestor entender que o relato } \\
\text { (problema) é relevante e encaminhar para } \\
\text { resolução, a reputação do usuário } \\
\text { aumenta. }\end{array}$ & 10 \\
\hline Colaboração & $\begin{array}{l}\text { A reputação do usuário aumenta a cada } \\
\text { novo usuário que registrar o mesmo } \\
\text { relato (problema). }\end{array}$ & 30 \\
\hline Adesão & $\begin{array}{l}\text { O usuário que registrou o relato } \\
\text { (problema) tem sua reputação ampliada a } \\
\text { cada “curtida” de outros usuários. }\end{array}$ & 20
\end{tabular}

A visibilidade que a reputação proporciona ao usuário se materializa através da "força"do relato. O cálculo da força do relato $F U V$ pode ser observado na equação (2).

$$
\mathrm{FUV}_{(\mathrm{r})}=\sum_{i=1}^{e_{i}} \frac{\left(v_{i} \cdot w_{i}\right)}{\max \left(v_{i}\right)}
$$

Onde $\left.F U V_{(} r\right)$ é a força do relato no app, $r$ identifica o relato, $e_{i}$ é a quantidade de elementos considerados no cálculo da força, $i$ é a variação de 1 até $e_{i}, v_{i}$ é valor de cada elemento (reputação, colaboração e adesão), $w_{i}$ é o peso de cada elemento e $\max \left(v_{i}\right)$ é maior valor de cada elemento.

O cálculo da força do relato considera os elementos apresentados na Tabela 2:

Tabela 2. Elementos que compõe a força de um relato.

\begin{tabular}{l|l|l}
\hline \multicolumn{1}{c|}{ Elemento } & \multicolumn{1}{|c}{ Descrição } & Peso \\
\hline Reputação & $\begin{array}{l}\text { A reputação do usuário que está relatando } \\
\text { um problema. }\end{array}$ & 40 \\
\hline Colaboração & $\begin{array}{l}\text { A quantidade de usuários que relataram o } \\
\text { mesmo problema. }\end{array}$ & 30 \\
\hline Adesão & $\begin{array}{l}\text { A quantidade de usuários que "curtiram" } \\
\text { o relato. }\end{array}$ & 30 \\
\hline
\end{tabular}

Os pesos da força do relato foram definidos de forma a garantir um equilíbrio entre os elementos que definem a força do relato, sendo a Reputação o mais importante e por consequência tendo um peso maior. 


\subsection{Algoritmo de Reputação}

A recomendação de encaminhamento para solução de problemas relatados é feita por meio do algoritmo descrito na figura abaixo.

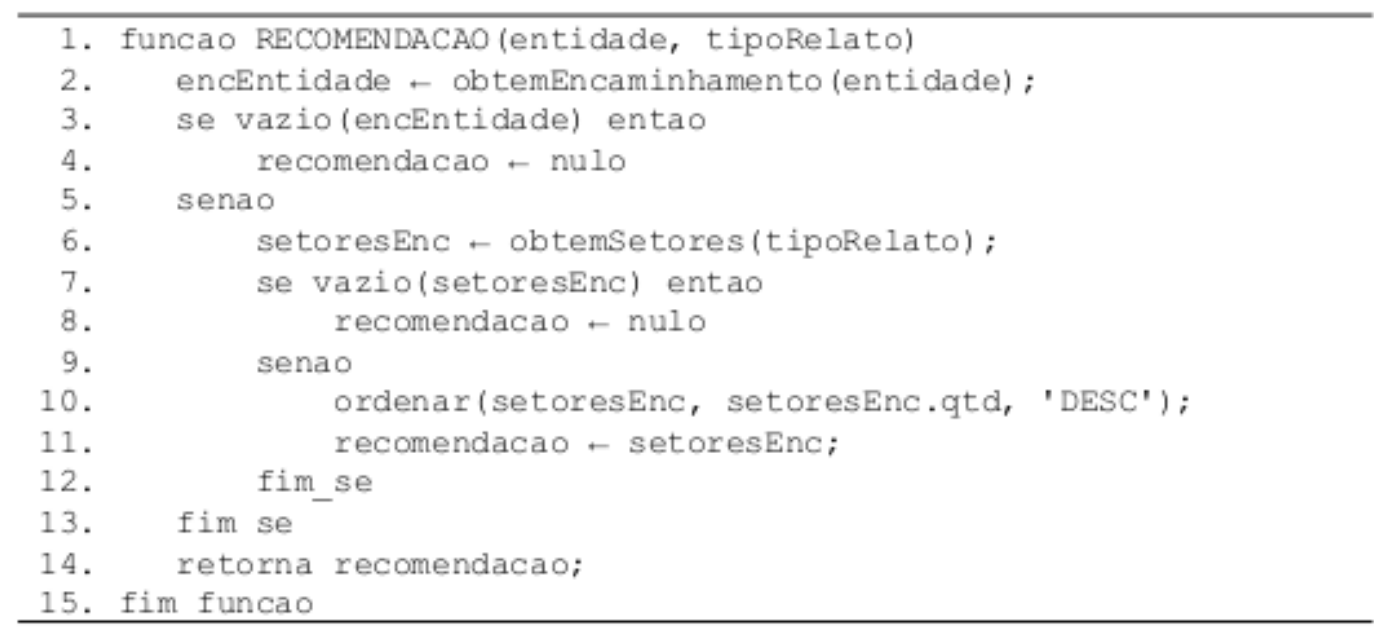

Figura 3. Pseudocódigo do algoritmo de recomendação.

Inicialmente, são obtidos todos os encaminhamentos de relatos feitos através de uma determinada entidade. A seguir, se houve retorno, são obtidos os setores para os quais estes encaminhamentos foram feitos e a quantidade de vezes que isso ocorreu. Após obter a lista de setores e a quantidade de encaminhamentos por setor, será feita a classificação em ordem numérica decrescente de quantidade de encaminhamentos. A lista de setores será então devolvida para o Painel de Gestão, onde será apresentada como sugestão para o gestor fazer um novo encaminhamento.

\section{Experimentos e Resultados}

Este seção apresenta os experimentos realizados. Para isto, apresenta-se uma introdução do público alvo utilizados no experimento, o primeiro com um baseline restrito e o segundo com um baseline mais abrangente.

$\mathrm{Na}$ aplicação dos experimentos, definiu-se inicialmente dois setores da Universidade de Passo Fundo que atuariam como Entidade Gestora, o público alvo e o período de execução dos experimentos. Então, o público alvo recebeu instruções por e-mail sobre o propósito do experimento, sobre como instalar o aplicativo e como colaborar, relatando problemas para o setor escolhido.

\subsection{Experimento 1}

O objetivo do Experimento 1 foi disponibilizar o aplicativo da abordagem Urban Voice a um grupo restrito e homogêneo de usuários, familiarizados com tecnologia, para que utilizassem todos os recursos da ferramenta, bem como encaminhassem ao autor as sugestões de melhorias e possíveis erros no aplicativo. Além disso, o experimento possibilitou que a Entidade Gestora utilizasse o painel de gestão para administrar os relatos dos usuários e também pudesse dar um feedback ao autor sobre melhorias e possíveis erros. 
O público alvo utilizado neste experimento, por ser mais restrito e ter como objetivo utilizar o aplicativo foram alunos, professores e funcionários vinculados à Área de Informática.

\subsection{Experimento 2}

O objetivo do Experimento 2 foi disponibilizar o aplicativo da abordagem Urban Voice a um grupo abrangente e heterogêneo de usuários, independentemente de domínio de tecnologia, para que utilizassem todos os recursos da ferramenta, bem como encaminhassem ao autor as sugestões de melhorias e possíveis erros no aplicativo.

Além disso, o experimento possibilitou que a Entidade Gestora utilizasse o painel de gestão para administrar os relatos dos usuários e também pudesse dar um feedback ao autor sobre melhorias e possíveis erros. Para o segundo experimento, a Entidade Gestora selecionada foi a Divisão Administrativa da UPF, que é responsável pela manutenção da infraestrutura do campus.

O público alvo deste experimento, por ser mais abrangente e ter como objetivo avaliar os mecanismos de reputação e recomendação, foram todos professores e funcionários da UPF.

\subsubsection{Análise dos Resultados}

Durante os experimentos foram recebidas 26 colaborações subjetivas de usuários por meio do e-mail que foi disponibilizado para feedback. Estas colaborações foram mensagens de apoio, apontamentos de erros e sugestões de melhorias na interface.

Durante os períodos em que o aplicativo e o painel de gestão estavam disponíveis para testes, todos os erros relatados foram corrigidos e as melhorias na interface foram implementadas. Em relação à adesão aos experimentos, pode-se observar uma baixa adesão dos usuários, ficando abaixo de 3\%. Pode-se afirmar que alguns fatores foram determinantes para esta baixa adesão: (i) $\mathrm{O}$ aplicativo desenvolvido somente para a plataforma Android, restringindo os usuários de iPhone; (ii) $\mathrm{O}$ pequeno prazo de duração do experimento; (iii) $\mathrm{O}$ formato da divulgação, por e-mail, restringiu o alcance.

Mesmo assim, os relatos inseridos pelos usuários foram importantes para testar o aplicativo e oportunizar ao gestor um mecanismo para que professores e funcionários pudessem relatar problemas de infraestrutura no campus da Universidade. Considera-se que o primeiro Experimento 1 obteve sucesso, pois a interface do usuário e os mecanismos de reputação e recomendação foram submetidos à prova com um grupo de usuários familiarizados com tecnologia e, como resultado, tanto o aplicativo quanto o Painel de Gestão foram aprimorados.

\section{Considerações Finais}

O objetivo deste artigo foi de apresentar uma abordagem baseada em perfil e recomendação, desenvolvida para dispositivos móveis, que oportuniza a usuários o apontamento de problemas de infraestrutura no contexto de smart campus, o acompanhamento da evolução da solução de problemas, bem como a interação com usuários que relataram os mesmos problemas. A abordagem também engloba um painel de gestão, administrado 
por uma entidade gestora, que recebe os relatos de problemas e encaminha as reclamações para os setores responsáveis pela solução.

Esta abordagem teve como premissa ser abrangente e adaptável. Abrangente, pois é possível utilizá-la em diferentes cenários e contextos, tais como em uma cidade, uma planta de uma fábrica, etc. Diferentemente de outras abordagens, a Entidade Gestora deve ser previamente cadastrada para que se possa utilizar o aplicativo, garantindo com isto o envolvimento desta.

Adaptável, pois os tipos de problemas que podem ser relatados são cadastrados pela Entidade Gestora, e desta forma é possível adaptar para novas situações que surgem. Por exemplo, é perfeitamente possível cadastrar um tipo de relato "Foco de Aedes Aegypti", cadastrar um setor para atender a esta demanda e passar a receber relatos. No desenvolvimento desta abordagem utilizou-se o perfil e a reputação do usuário como elementos estruturantes da recomendação. Pois a reputação do usuário, obtida através da sua participação no Urban Voice, somada com a participação de outros usuários, ao curtir ou aderir a determinado relato, geram a "força"do relato. Esta força recomenda para a entidade Gestora qual relato deve dar atenção, destacando-o no Painel de Gestão.

A recomendação também é usada no momento que o gestor vai encaminhar o relato para um setor. $\mathrm{O}$ algoritmo de recomendação avalia os dados históricos e oferece como sugestão encaminhar para um setor que já tenha atendido aquele tipo de relato anteriormente. Os experimentos realizados foram valiosos para evoluir o Aplicativo e o Painel de Gestão, removendo erros e aprimorando a interface.

Através dos resultados destes experimentos pode-se constatar que a abordagem cumpre o seu papel de ser um canal de comunicação entre o usuário e a Entidade Gestora e, por consequência, traz o empoderamento de seus usuários. Porém, observou-se que é fundamental que a Entidade Gestora esteja comprometida e seja ágil no atendimento dos relatos para que estes sejam resolvidos. Além dessas melhorias, pretende-se evoluir o aplicativo e expandir sua utilização fora do contexto de smart campus, na cidade de Passo Fundo.

\section{Referências}

Abuein, Q., Shatnawi, A., and Al-Sheyab, H. (2017). Trusted recomendation system based on level of trust (trs-lot). In 2017 International Conference on Engineering and Technology (ICET), pages 1-5.

Abuelyaman, E. S. (2008). Making a smart campus in saudi arabia. Educause Quarterly, 31(2):10-12.

Albino, V., Berardi, U., and Dangelico, R. M. (2015). Smart cities: Definitions, dimensions, performance, and initiatives. Journal of urban technology, 22(1):3-21.

Calegari, S. and Pasi, G. (2010). Ontology-based information behaviour to improve web search. Future Internet, 2(4):533-558.

Capdevila, I. and Zarlenga, M. I. (2015). Smart city or smart citizens? the barcelona case. Journal of Strategy and Management, 8(3):266-282. 
Eremia, M., Toma, L., and Sanduleac, M. (2017). The smart city concept in the 21 st century. Procedia Engineering, 181:12 - 19. 10th International Conference Interdisciplinarity in Engineering, INTER-ENG 2016, 6-7 October 2016, Tirgu Mures, Romania.

Flôr, C. and Teixeira, C. (2018). Intelligent and entrepreneurship cities: A comparative study between rankings. International Congress of Knowledge and Innovation - Ciki, $1(1)$.

Hirsch, B. and Ng, J. W. (2011). Education beyond the cloud: Anytime-anywhere learning in a smart campus environment. In 2011 International Conference for Internet Technology and Secured Transactions, pages 718-723. IEEE.

Lopes, G. R. (2012). Avaliação e recomendação de colaborações em redes sociais acadêmicas.

Loriot, M., Aljer, A., and Shahrour, I. (2017). Analysis of the use of lorawan technology in a large-scale smart city demonstrator. In 2017 Sensors Networks Smart and Emerging Technologies (SENSET), pages 1-4.

Mirri, S., Roccetti, M., and Salomoni, P. (2018). Collaborative design of software applications: the role of users. Human-centric Computing and Information Sciences, $8(1): 6$.

Muhamad, W., Kurniawan, N. B., Yazid, S., et al. (2017). Smart campus features, technologies, and applications: A systematic literature review. In 2017 International Conference on Information Technology Systems and Innovation (ICITSI), pages 384391. IEEE.

Prandi, C., Monti, L., Ceccarini, C., and Salomoni, P. (2019). Smart campus: Fostering the community awareness through an intelligent environment. Mobile Networks and Applications.

Prandi, C., Nisi, V., and Nunes, N. (2018). On exploring a pervasive infrastructure to foster citizens participation and sustainable development. In Proceedings of the 32nd International BCS Human Computer Interaction Conference, page 223. BCS Learning \& Development Ltd.

Tse, R., Xiao, Y., Pau, G., Fdida, S., Roccetti, M., and Marfia, G. (2016). Sensing pollution on online social networks: A transportation perspective. Mobile Networks and Applications, 21(4):688-707.

Yu, Z., Liang, Y., Xu, B., Yang, Y., and Guo, B. (2011). Towards a smart campus with mobile social networking. In 2011 International Conference on Internet of Things and 4th International Conference on Cyber, Physical and Social Computing, pages 162169. IEEE.

Özcan, U., Arslan, A., İlkyaz, M., and Karaarslan, E. (2017). An augmented reality application for smart campus urbanization: Msku campus prototype. In 2017 5th International Istanbul Smart Grid and Cities Congress and Fair (ICSG), pages 100-104. 